\title{
16 Notfallseelsorge als Hilfe zur Bewältigung
}

\author{
Christoph Pompe
}

\subsection{Strukturen der Notfallseelsorge}

Seelsorge in Situationen erlebter extremer Not ist in den christlichen Kirchen und Gemeinden seit Beginn ihrer Existenz ausgeübt worden. Sobald angesichts des jähen, unvorhersehbaren Todes Hilfe nötig wurde, gab es die Bereitschaft, diese auszuüben. Der Apostel Paulus erlebt (Apostelgeschichte 20, 7-12) den Fenstersturz seines Zuhörers Eutychus, der zunächst für tot gehalten wird. Paulus umarmt diesen, behauptet wider den ersten Eindruck dessen Leben. Dies erweist sich als wahr - und die Anwesenden „wurden über die Maßen getröstet“ (Apostelgeschichte 20, Vers 12). Schon früh zeichnen sich Grundprinzipien der Notfallseelsorge ab: Präsenz und Trost im Angesicht extremen Erlebens sind die wesentlichen Wirkfaktoren.

Im Angesicht des zu erwartenden Todes entstand der Ritus der Krankensalbung (Sakrament in der römisch-katholischen Kirche), nach Eintritt des Todes war die „Aussegnung “ in den protestantischen Gemeinden, die „Verabschiedung“ in der katholischen Kirche ritueller Brauch.

Notfallseelsorge als eigene fachliche Disziplin und als organisatorisch gegliederter Teil der psychosozialen Notfallversorgung (PSNV) entstand aufgrund der Initiative von Pfarrerinnen und Pfarrern in der evangelischen und katholischen Kirche in Deutschland (aber auch in Österreich, Schweiz, Luxemburg) Anfang der Neunziger Jahre des 20. Jahrhunderts - als „bottom-up“-Bewegung. In den siebziger Jahren waren „Top-down“-Überlegungen wie der „Unfallfolgedienst“ für Versorgung von Opfern von Verkehrsunfällen aus deutschen Autobahnen entstanden, aber nie zu Realisierung gediehen. 
In den nunmehr 25 Jahren der Notfallseelsorge sind verlässliche Strukturen der Qualitätssicherung in Form von Ausbildungsstandards definiert worden, öffentliche Aufmerksamkeit und Anerkennung (z.B. in der Berichterstattung bei Croßschadensereignissen) ist Routine geworden.

Zwei Elemente unterscheiden Notfallseelsorge von fachlich vergleichbaren anderen Fachangeboten (z.B. Kriseninterventionsteams [KIT]; Kriseninterventionsdienst [KID], die in den Hilfsorganisationen Johanniter Unfallhilfe, Malteser Hilfsdienst, Arbeitersamariterbund, Deutsches Rotes Kreuz bereit gehalten werden):

- Notfallseelsorge geschieht in ausdrücklicher Beauftragung durch die christlichen Kirchen, die in der „Arbeitsgemeinschaft Christlicher Kirchen in Deutschland (ACK)“ zusammengeschlossen sind;

- Notfallseelsorge ist grundsätzlich ehrenamtliche, nachweisbar qualifizierte Tätigkeit. Vielerorts werden Regiekosten (Ausrüstung, Fahrtkosten, Öffentlichkeitsarbeit, Supervision usw.) als freiwillige Leistung aus öffentlichen und kirchlichen Etats wie auch aus Spenden- und Kollektenmitteln aufgebracht. Sachleistungen werden häufig durch die genannten Hilfsorganisationen unterstützt.

Für Opfer, Zeugen und Angehörige ist die Leistung der Notfallseelsorge stets kostenlos.

Von den übrigen „Sonderseelsorgebereichen“ der Kirchen (z.B. Krankenhausseelsorge, alle Formen der Kategorialseelsorge für spezifische Cruppen) ist die Notfallseelsorge deutlich unterschieden: sie ist eng an die Alarmierungswege der regionalen Leitstellen des Rettungsdienstes gebunden, angewiesen auf Anfragen aus den polizeilichen und notärztlichen Arbeitsbereichen - nicht wie sonst in der Seelsorge üblich auf direkte Nachfrage durch die Betroffenen.

Eine weitere Besonderheit liegt in ihrem streng subsidiären Charakter: in aller Regel ist ein Notfallseelsorgeeinsatz einmalig, allenfalls mit Weiterempfehlung zu Diensten der „Zweiten Linie“ wie psychologischen Beratungsstellen, gemeindlicher Seelsorge, fachärztlicher Behandlung, psychotherapeutischer Praxis oder Selbsthilfegruppen.

\section{Merke:}

\section{Notfallseelsorge: qualifizierte Seelsorge in Extremsituationen \\ - im kirchlichen Auftrag \\ - ehrenamtlich ausgeübt \\ - eingebunden in rettungsdienstliche Strukturen}

Die ersten Notfallseelsorgeeinsätze wurden Anfang der neunziger Jahre von Pfarrern mit engen Verbindungen zu den in ihren Gemeinden tätigen Feuerwehren durchgeführt. Sie erlebten unmittelbar die dienstlichen Belastungen der Einsatzkräfte und waren als Seelsorger für diese und von diesen gefragt. Seit diesen ersten Anfängen waren stets die beiden Einsatzbereich „Hilfe für belastete Einsatzkräfte“ und „Hilfen für Opfer, Zeugen und Angehörige“ eng verbunden. Standards für beide fachlichen Leistungen waren zunächst nicht vorhanden. Wissenschaftliche Begleitung dieser fachlichen Leistungen fehlten zunächst weitgehend. Aus der überregionalen Zusammen- 
arbeit der „Feuerwehrseelsorge“ und deren Arbeitsgemeinschaft wurde am 16. März 1998 die „Konferenz evangelischer Notfallseelsorger und Notfallseelsorgerinnen“ auf nationaler Ebene gegründet - mit der Verabschiedung der „Kasseler Thesen“, in denen erstmalig die beiden genannten Aufgabenbereiche und Zielgruppen (Opfer, Zeugen Angehörige einerseits und belastete Einsatzkräfte andererseits) definiert wurden.

Auch mit den einschneidenden Einsatzerfahrungen von Großschadenslagen wie z.B. dem ICE-Unglück in Eschede 1998 wurde eine Aktualisierung der Grundlagen mit den „Hamburger Thesen“ der Notfallseelsorge am 12. September 2007 möglich und notwendig (Müller-Lange et al. 2013: 23-27). Selbstverständnis, Auftrag und Qualitätsstrukturen fanden auch außerhalb des Bereiches der verfassten evangelischen und katholischen Kirche Beachtung: seit 2002 wird für die Unterstützung von Betroffenen und Einsatzkräften die Bezeichnung „Psychosoziale Notfallversorgung (PSNV)“ benutzt. Das Bundesamt für Bevölkerungsschutz und Katastrophenhilfe (BBK) hatte seit diesem Zeitpunkt Forschungsprojekte beauftragt und den sogenannten „Konsensus-Prozess“ zur Qualitätssicherung im Bereich PSNV initiiert (Müller-Lange et al. 2013). Eine Vielzahl von staatlichen und kirchlichen Einrichtungen (wie die Notfallseelsorgegremien der evangelischen und katholischen Kirchen) wie auch Fachverbänden (wie z.B. der Deutsche Feuerwehrverband, die Hilfsorganisationen u.a.) haben die Ergebnisse dieses Konsensus-Prozesses 2011 einvernehmlich verabschiedet, u.a. eine einheitliche Terminologie definiert und Qualitätsmindeststandards für Aus- und Fortbildung von Fachkräften der PSNV vereinbart.

Für die Notfallseelsorge ist ihre Einbindung in die traditionelle Gefahrenabwehr bei komplexen Gefahren- oder Schadenslagen, aber insbesondere die Integration ihres klassischen Aufgabengebietes zur Bewältigung individueller Notfälle (bei Verkehrsunfälle, plötzlichen Todesfällen im häuslichen Bereich usw.) in das Gesamt aller PSNV-Angebote essentiell. Damit ist Notfallseelsorge anerkannter Teil der öffentlichen Daseinsfürsorge.

Außerdem wird neben der geschilderten Erstversorgung für Opfer, Zeugen und Angehörige nach extremem Erleben mit davon unterschiedener fachlicher Expertise die psychosoziale Unterstützung von sämtlichen Einsatzkräften in Prävention, Intervention und Postvention nach besonders belastenden Einsätzen geregelt.

Hintergrund waren die ausgewerteten Erfahrungen in der Bewältigung großer Schadensereignisse (wie Flugschauunfall Ramstein 1988, ICE-Unglück Eschede 1998, Tsunami-Katastrophe 2004, Love-Parade-Unglück Duisburg 2010).

Die Hilfsorganisationen (Arbeiter-Samariter-Bund, Deutsches Rotes Kreuz, Malteser Hilfsdienst, Johanniter Unfall-Hilfe) wie auch die Konferenz evangelischer Notfallseelsorge und die Konferenz der Diözesanbeauftragten für die Katholische Notfallseelsorge haben gegenseitige Anerkennung der Crundlagen im Bereich der Psychosozialen Akuthilfen wie auch der Aus- und Fortbildung im Bereich der Psychosozialen Akuthilfen im Jahr 2013 beschlossen. Ein Mindestumfang der fachlichen Fortbildung von 8 o Unterrichtseinheiten ist seitdem vereinbart.

Diese Mindeststandards werden im kirchlichen Bereich erweitert, z.B. mit Elementen des liturgischen Handelns und der theologischen Grundlagen der Notfallseelsorge.

Freiwilligkeit der Inanspruchnahme von jeder Form von Psychosozialer Akuthilfe durch Zeugen, Opfer und Angehörige und Kostenlosigkeit der Einsätze für diesen Nutzerkreis sind ebenfalls Teil der einvernehmlichen Standards. 
Notfallseelsorge geschieht in der Regel als unentgeltliche Leistung - sei es durch ehrenamtlich tätige Fachkräfte, sei es durch hauptamtliche Geistliche im Rahmen ihres verabredeten pastoralen Dienstauftrages.

\section{Merke: \\ - Inanspruchnahme von Notfallseelsorge immer freiwillig \\ - Keine Behandlung von Störungen mit Krankheitswert \\ - Verbindlich abgesprochene Standards in Deutschland \\ - Notfallseelsorge ist keine missionarische Tätigkeit}

Wesentliche fachliche Grundlagen aller Psychosozialen Akuthilfen betreffen Indikation, Ausschlusskriterien und Abgrenzung von heilkundlichen Leistungen. So sind auch bei Notfallseelsorgeeinsätzen

- akutpsychiatrische Krisen

- akute Suizidalität (mit sog. „talk down“ durch andere Fachdienste)

- Deeskalation im Rahmen polizeilicher Lagen (wie Geiselnahme)

ausgeschlossen und bedürfen ggf. fachärztlicher, psychotherapeutischer oder polizeilicher Intervention.

Notfallseelsorge ist stets und überall in Deutschland ökumenisch wahrgenommene Aufgabe: sie geschieht im Auftrag der entsendenden Kirchen und Gemeinden, die in den regionalen Arbeitsgemeinschaften Christlicher Kirchen in Deutschland (ACK) zusammengeschlossen sind.

Unabhängig von dieser Beauftragung durch christliche Kirchen und Gemeinden ist die Zielrichtung immer die Offenheit für alle Menschen in Extremsituationen, die diese Hilfe wünschen - unabhängig davon, ob sie einer Claubensgemeinschaft zugehörig sind oder nicht. Eine konfessionelle „Passung“ von Notfallseelsorger und Angehörigen wäre allein wegen der Alarmierungslogistik nicht möglich. So können im Einsatz Menschen ganz verschiedener Glaubensrichtungen aufeinander treffen. Die Auswertung der Einsätze ergibt aber: dies bedeutet in der Regel kein Problem. Allerdings wird ein Notfallseelsorger/eine Notfallseelsorgerin bei der Erkundung von womöglich hilfreichen Ressourcen von Opfern, Zeugen und Angehörigen aufmerksam sein für glaubensmäßige oder gemeindliche Bezüge und die Weiterleitung zu den möglichen Formen von weiter begleitender Seelsorge in den je eigenen Denominationen. Notfallseelsorge ist keine Form der Mission für die eigene Glaubensrichtung. Sie ist als Dienst der „Ersten Linie“ strikt subsidiär zu den Diensten der „Zweiten Linie“, zu denen z.B. klassische Angebote der Seelsorge der Denominationen zählen. Bei Einsätzen in muslimischen Familien gilt es, alles Nötige für die hier nach den Lehren des Islam notwendigen Hilfen durch die Großfamilie, die Gemeinschaft der muslimischen Gläubigen und ggf. den zuständigen Imam der eigenen Moscheengemeinde zu unterstützen. In Freikirchen gibt es häufig für Seelsorge besonders beauftragte und befähigte Gemeindeglieder und Laien, an die durch die Notfallseelsorge weitergeleitet werden soll.

Soll solche Weiterleitung gelingen, bedarf es umfänglicher Vertrautheit mit der in Deutschland vorfindlichen Vielfalt religiösen Lebens, Einbindung in das lokale System der Notfallseelsorge und Kenntnis der lokalen Bedingungen. 
Bei großen Schadensereignissen gelten in der Notfallseelsorge ähnliche Prinzipien des Einsatzaufbaus, der Aufteilung in Einsatzabschnitte, der Führung und der Nachalarmierung, exakt so, wie sie in den übrigen Fachdiensten des Rettungswesens bewährt sind. Sie werden in der Regel in Übungen auf regionaler Ebene immer wieder erprobt. Beim Love-Parade-Unglück 2010 in Duisburg waren am Schadenstag 9o Einsatzkräfte der Notfallseelsorge - nachalarmiert aus den umliegenden Regionen Nordrhein-Westfalens - tätig. Komplexe Aufgaben wie Medienarbeit, umfängliche Nachsorgemaßnahmen, Mitwirkung an speziell auf solche Großschadenslagen bezogenen Gottesdienste, geordnete Ablösung von Einsatzkräften: all dies war und ist nur möglich in überörtlicher Absprache nach vorher abgesprochenen Algorithmen. In einigen Bundesländern gibt es bereits heute landesweite Leitungsstrukturen der Psychosozialen Notfallversorgung, die bei großen Schadensfällen aktiviert werden und helfen, die Notfallseelsorge mit den übrigen Hilfsangeboten zu koordinieren.

\subsection{Aufgabe der NFS bei Betroffenen und bei den Fachkräften der Hilfsinterventionen}

\subsubsection{Aufgaben bei Betroffenen}

Eine Unfallstelle absichern, Verletzte bergen, ausgelaufenen Treibstoff abbinden, Löscharbeiten: die Aufgaben der Feuerwehr bei einem Verkehrsunfall sind klar definiert, ebenso die rettungsdienstlichen und notärztlichen Handlungsnotwendigkeiten: Kreislauf- und Atmungsstabilisierung, Blutstillung, Reanimationsmaßnahmen usw. Die Aufgaben der Notfallseelsorge sind zunächst schwieriger zu beschreiben. So sprechen die „Hamburger Thesen“ (verabschiedet von der Konferenz evangelischer Notfallseelsorge 2007) von der „Präsenz des Seelsorgers, der Seelsorgerin vor Ort und dem Angebot einer helfenden Begleitung in der Akutphase“ (Müller-Lange et al. 2013).

„Präsenz“ und „hilfreiche Begleitung in Akutphase“: beides trifft im Grunde für alle professionellen Helferinnen und Helfer in Extremsituationen ebenfalls zu. Die anderen Professionen haben aber neben der basalen Form von Zuwendung auch andere fachliche Aufgaben. In aller Regel ist die basale menschliche Zuwendung durch die fachgerechte Erledigung dieser übrigen Aufgaben und ein begrenztes Zeitbudget eingeschränkt. Notfallseelsorge hat in aller Regel genügend Zeit zur Verfügung, um zumindest bis zu einem gewissen Abschluss der Akutphase präsent zu sein. Sie entstand paradoxerweise aus dem zunächst völligen Fehlen jeder geistlichen Präsenz durch die Professionalisierung der Rettungsdienste: ab den sechziger Jahren ist die Anwesenheit von Geistlichen an Unglücksorten nur noch zufällig möglich, da Bergung und Abtransport in Verbindung mit lebensrettenden Sofortmaßnahmen und wachsenden Möglichkeiten der intensivmedizinischen Versorgung auch während der Transporte (z.B. Luftrettung) stattfinden. Helfende Intervention in Gestalt von Gebet, Aussegnung und anderen Ritualen ist im Abstand von Zeit und Raum nicht möglich.

Empirische Studien zur Wirksamkeit von „Seelischer Erster Hilfe (Lasogga u. Gasch 2009) belegen, dass Opfer, Zeugen und Angehörige in erster Linie die verlässliche Anwesenheit von Ersthelferinnen/Ersthelfern als wesentliche Hilfe beschreiben - und post hoc insbesondere deren Fehlen als zusätzlichen erheblichen Belastungsfaktor nach Unglücken erinnern, wenn sie nach Schadensfällen befragt werden. Fehlt sol- 
che Präsenz, steigert sich unmittelbar das Gefühl von Verlassenheit und bedrohlicher Verwirrung. Seelische Hilfe ist wirksam, wenn sie

- möglichst schnell nach dem Schadensereignis angeboten wird,

v wenn sie ohne Unterbrechung geschieht und genügend lange andauert.

Diese Hilfe ist mit wenigen Grundregeln auch von zufällig am Schadensort anwesenden Laien leistbar, wenn diese sich als Helfende namentlich vorstellen, wenn sie glaubhaft versichern, dass Rettung bald kommt, wenn sie vor Zuschauenden abschirmen und vorsichtigen Körperkontakt zum Unfallopfer aufnehmen („4-S-Regel“ Lasogga u. Gasch 2009).

Ein Fallbeispiel für solche basale, wirksame Hilfe aus dem Bereich „häuslicher Todesfälle“ wird hier aufgeführt:

20:15 Ehemann (70) kollabiert im Badezimmer.

20:17 Ehefrau (71) alarmiert telefonisch die Leitstelle des Rettungsdienstes und erhält dort Instruktionen zu lebensrettenden Maßnahmen.

20:23 Innerhalb des angestrebten Zeitfensters sind Notärztin und Rettungsassistenten am Einsatzort und setzen die Reanimationsmaßnahmen der Ehefrau fort. Ein Rettungsassistent schlägt vor (da die Ehefrau mehr und mehr schluchzt), die Notfallseelsorge nachzualarmieren, das Rettungsteam berät dies kurz, stimmt einvernehmlich zu - die Anforderung geht telefonisch an die Leitstelle. Die Prognose für den Ehemann scheint ungünstig.

20:45 Leitstelle alarmiert den diensttuenden Notfallseelsorger. Das Rettungsteam setzt die Reanimation in Erwartung des Notfallseelsorgers fort, auch wenn die Aussicht auf Erfolg der Maßnahme schwindet.

21:10 Der Notfallseelsorger trifft in der Wohnung ein, die Notärztin teilt ihm in der Türöffnung schon mit, dass die Rettungsmaßnahmen leider eingestellt werden - mangels Aussicht auf Einsetzen der Herztätigkeit. Der Notfallseelsorger stellt sich er Ehefrau vor und bittet sie, mit ihr im Wohnzimmer Platz zu nehmen.

21:25 Das Rettungsteam stellt die Ordnung in der Umgebung des Leichnams in etwa wieder her, die Notärztin füllt die Todesbescheinigung aus, Das Team kondoliert bei der Witwe, verabschiedet sich vom Notfallseelsorger und rückt aus. Der Notfallseelsorger bleibt, spricht mit der Witwe über die letzten gemeinsamen Tage, fragt behutsam, ob der Tod als mögliches Ereignis jemals in den Gesprächen des Ehepaares vorgekommen sei. Er erkundigt sich nach Kindern, Angehörigen, bietet seine Hilfe beim Kontakt zum Bestatter an.

22:00 Der Sohn des Verstorbenen trifft ein - telefonisch informiert. Der Notfallseelsorger hat verstanden, dass ein loser Kontakt zu einem örtlichen Pfarrer bestanden hat - er wird diesen am Morgen des folgenden Tages informieren. Er bietet Gebet und Segen am Sterbebett an, die Witwe und der Sohn sind einverstanden. Der Notfallseelsorger zündet eine Kerze neben dem Bett an, betet den traditionellen „Valetsegen“, bittet Gott um Beistand für Sohn, Ehefrau und alle übrigen Trauernden.

22:40 Der gewünschte Bestatter trifft ein, der Notfallseelsorger vergewissert sich, dass seine Anwesenheit nun nicht mehr erforderlich ist und verabschiedet sich.

23:05 Notfallseelsorger meldet Leitstelle: „Zurück vom Einsatz“. 
Für Außenstehende ist dies sicher eine wenig spektakuläre Standardsituation - aus den Rückmeldungen aus den nachfolgenden Trauergesprächen der gemeindlichen Seelsorge wird deutlich, dass die Erstintervention als erheblich Stärkung für die Angehörigen erlebt wird. Die Besonderheit der Einbeziehung der Betroffenen (Polizist: „Möchten Sie mit jemandem von der Notfallseelsorge sprechen?“), die Orientierung auf vorhandene Ressourcen der Bewältigung (Angehörige, Nachbarn, religiöse Bindungen), die zeitliche Flexibilität bis zur Übergabe an Bestatter oder eintreffende Freunde und Angehörige: all dies macht deutlich, dass „Präsenz und hilfreiche Begleitung" faktisch aufwendige und komplexe Leistungen sind, die professionelles Handeln erfordern. Zudem steht als Systemleistung im Hintergrund ein regionales, qualifiziertes und nachalarmierbares Netz von anderen Fachkräften bereit - z.B. mit Fremdsprachenkenntnissen oder als qualifizierte muslimische Notfallbegleitung. Verlässlichkeit der Anfahrt auch bei extremen Wetterlagen durch Transporthilfe der Feuerwehren, geregelte Ablösung bei langen Einsätzen, eigene Supervision der Einsätze, anonymisierte Dokumentation sind Teil der Qualitätssicherung.

Die Mehrzahl der Einsätze der Notfallseelsorge (ca. 65\%/Schätzwert) sind vergleichbare Einsätze im häuslichen Bereich. Die übrigen Einsätze im außerhäuslichen Bereich modifizieren die Grundprinzipien notfallseelsorgerlichen Handelns dadurch, dass Ressourcen außerhalb des Wohnumfelds schwerer ausfindig zu machen sind, dass ggf. Gesprächsführung im Freien oder im Einsatzfahrzeug stattfinden muss, das die Exposition von Opfern, Zeugen und Angehörigen vor „Gaffern“ und Medienvertretern eine erhebliche Zusatzbelastung darstellen kann. Die Notfallseelsorge sieht ihre Aufgabe auch im Schutz gegenüber diesen Komplikationen.

NFS als „Dienst der ersten Linie“ setzt voraus, dass fachlich qualifizierte „Dienste der zweiten Linie“ wie Beratungsstelle, Psychotherapeuten/innen, gemeindliche Seelsorge, Selbsthilfegruppe, Akutpsychiatrische Hilfen bereitstehen. Die Akuthilfe für Betroffenen besteht in der Kompetenz der NFS, eine vorläufige Expertise im Sinne von Erstscreening vorzunehmen, ohne umfängliche sachkundige psychodiagnostische Leistungen erbringen zu müssen. In der Ausbildung zu NFS werden die einschlägigen Störungsbilder wie „Akute Belastungsreaktion (ICD 10 F43.o)“ unterrichtet - um im Einsatz kritische Entwicklungen von der angemessenen Trauerreaktion unterscheiden zu können und ggf. Weiterempfehlungen geben zu können. Auch weitere Traumafolgestörungen wie Posttraumatische Belastungsstörung (ICD 10 F43.1) sind Teil der Fortbildungseinheiten zu Psychotraumatologie, führen aber in der Akutsituation nicht zu einem eigenständigen heilkundlichen Handeln der NFS. Die Nähe zum Schadensereignis würde im Übrigen auch keine sachgerechten Diagnosestellungen oder gar traumatherapeutisches Handeln für Fachkräfte ermöglichen. Im Einsatzalltag ist die Schnittstelle zu notärztlichem Handeln wichtig: wann ist medikamentöse Sedierung bei heftiger Erregung indiziert, wann verhindert diese schnelle Sedierung das seelsorgerliche Gespräch?

Lösbar sind solche schwierigen Entscheidungen und Einschätzungen, wenn die genauen Kenntnisse der je anderen Professionalität in Medizin und Seelsorge mit Wertschätzung auf Basis von als gelungen erlebten gemeinsamen Einsatzlagen besteht. 


\subsubsection{Aufgaben der NFS bei den Fachkräften der Hilfsintervention}

Diese bestehen zunächst in deren Entlastung von Doppelaufgaben in Bezug auf Zeugen und Angehörige (Feuerwehrmann: „Die NFS hält mir beim Verkehrsunfall den Rücken frei.“). Rettungsdienste arbeiten unter hoher Stressbelastung - in der Regel müssen sie ohne Verzug nach Erledigung der Kernaufgaben zurück in den Standort, um für weitere Alarmierung bereit zu stehen. Lange Verweildauer am Schadensort ist kaum möglich. Gerade der Abbruch von Reanimationsmaßnahmen, die ohne Erfolg bleiben, kann die Fachkräfte enorm belasten. Eine von Vertrauen getragene Übergabe der Betreuung von Angehörigen an die NFS entlastet - kann die NFS doch ggf. auch einige Stunden am Einsatzort bleiben. Ähnliche gilt für die Kooperation mit Polizeikräften: die Überbringung von Todesnachrichten ist als hoheitliches Handeln Aufgabe der Polizei. NFS ist Anfang der neunziger Jahre aus den Erfahrungen auch der Polizeiseelsorge entstanden. Die Begleitung von Polizeibeamten/innen bei der Überbringung von Todesnachrichten ist seitdem eine bewährte gemeinsame Aufgabe von Polizei und NFS. Dies schlägt sich in den Leitlinien der Innenministerien nieder, die die Kooperation empfehlen. Professionelle Kooperation setzt eine genaue gegenseitige Kenntnis der Rollenprofile der andern Fachkräfte voraus. In der Fortbildung zur Mitarbeit in der NFS wird dies u.a. in Hospitationen bei Feuerwehr und im Wachund Wechseldienst der Polizei gelernt. In den Aus- und Fortbildungen der Feuerwehr, den rettungsdienstlichen Fortbildungen und den Polizeiausbildungen wiederum wird über die Rollenprofile der NFS informiert. Im Einsatz nicht verhandelbare Grundprinzipien des jeweiligen Handelns (wie Ermittlungsnotwendigkeit der Polizei, Vorrang lebensrettender Maßnahmen im rettungsdienstlichen Handeln, seelsorgerliche Verschwiegenheit bei NFS) können zu Belastungen der Kooperation führen.

Verbindliche Routinen, die regelmäßig gemeinsam geübt werden, die Einordnung der NFS in Struktur und Leitung des Einsatzes (Polizei, Feuerwehr oder ärztlicher Leiter Rettungsdienst), definierte Einsatzstichworte für verbindliche Mitalarmierung der NFS (z.B. „Todesnachricht überbringen“) und regional abgesprochene Wege der Dokumentation und Umgang mit Beschwerden verbessern das wachsende kollegiale Vertrauen in die jeweilige Kompetenz des Anderen.

Einsatzkräfte sind oft Opfer von Projektion und Vorwürfen durch Opfer, Zeugen und Angehörige - bis hin zu Gewalthandlungen. Im günstigsten Fall hat die NFS hier eine vermittelnde Position zwischen den Beteiligten. Besonders deutlich wird dies im Fall von Plötzlichem Kindstod (SID):

\section{Ein Fallbeispiel:}

Die Eltern finden ihren dreimonatigen Sohn morgens leblos im Kinderbett vor. Sie alarmieren den Rettungsdienst. Die wenigen Minuten bis zum Eintreffen des Notarztwagens dehnen sich in der Wahrnehmung der Eltern. Sie ertragen dies kaum. Die Notärztin stellt nach Untersuchung den Tod des Kindes fest und erklärt den Eltern, dass der Tod wahrscheinlich vor einigen Stunden eingetreten ist und sie deshalb keine Reanimationsmaßnahmen durchführen wird. Die Anamnese ergibt keine Informationen über Vorerkrankung. Die Notärztin bietet die Alarmierung der NFS an, was die Eltern akzeptieren. Auf der Todesbescheinigung kreuzt sie an, dass die Todesursache ungeklärt ist. Dies hat die Nachalarmierung des kriminaltechnischen Dienstes zur Folge. Die Polizeibeamten treffen - gleichzeitig mit dem NFS ein. Der Notfallseelsorger bittet die Eltern zum Gespräch in das Wohnzimmer - die Polizei- 
beamten schließen zur Untersuchung des Kindes die Zimmertür. Sie prüfen u.a., ob Spuren von Fremdeinwirkung an der Leiche zu finden sind. Währenddessen spricht der Notfallseelsorger mit den Eltern auch über die Beschlagnahme des Kindes, eine weitere, für die Eltern stets unerwartete Extrembelastung. Sind doch die Möglichkeiten eines Abschieds von dem Kind in aller Ruhe nun nicht gegeben.

Rechtliche Notwendigkeiten stehen offenbar gegen seelsorgerliche Erfordernisse. In der Auswertung solcher Einsätze bestätigen die Polizeibeamten/innen oft, dass sie auch unter den rechtlichen Erfordernissen leiden und die vermittelnden Rolle der NFS schätzen.

In den „Hamburger Thesen“ 2007 (Müller-Lange et al. 2013: 26) wird als besonderes Arbeitsfeld der NFS die Seelsorge in Feuerwehr, Rettungsdienst und Katastrophenschutz benannt. Besondere Belastungen im Einsatz, Mitarbeit der NFS in Aus- und Fortbildung, Gottesdienste und Amtshandlungen, Beratung der Organisationen im Bereich PSNV und Beratung der Einsatzleitung bei Einsätzen: für diese Aufgaben sind besondere Qualifikationen und Aufträge notwendig. Hier ergeben sich die meisten fachlichen Überschneidungen mit den internen Ressourcen der Hilfsorganisationen, des THW, der Polizei, der Verkehrsunternehmen und der Bundeswehr, die diese für ihre Einsatzkräfte vorhalten. Am häufigsten ist die Beauftragung der Feuerwehrseelsorge: „Fachberater Seelsorge“ (in der Nomenklatur der Wehren) werden durch die Wehrführung mit Zustimmung einer Kirche ernannt und sind Mitglieder ihrer Wehr. Grundsätzlich gilt: Einsatznachsorge ist Aufgabe der jeweiligen Dienstgeber. In den vielfältigen fachlichen Angeboten (z.B. Organsisierte Personalunterstützung für Extremeinsätze und Nachsorge (OPEN), Critical Incident Stress Management (CISM) nach J.T. Mitchell) wird in aller Regel mit dem Konzept der Entlastung durch besonders trainierte Peers in Verbindung mit psychosozialen Fachkräften gearbeitet.

\subsection{Qualifikation für Notfallseelsorger/innen}

Notfallseelsorge ist „Grundbestandteil des Seelsorgeauftrages der Kirchen“- so in den „Hamburger Thesen“ 2007 beschrieben (Müller-Lange et al. 2013: 25). Nach den Pionierjahren Anfang der neunziger Jahre wurden nach und nach bewährte und unverzichtbare Ausbildungselemente erprobt, ab 2002 durch Forschungsaufträge seitens des Bundesamtes für Bevölkerungsschutz und Katastrophenhilfe (BBK) evaluiert. „Psychosoziale Akuthilfe“ ist seit 2011 der Oberbegriff für das Gesamt der Angebote in kirchlichen und weltlichen Organisationen, für die es im Rahmen des „Konsensus-Prozesses“ gelang, im Einvernehmen aller einschlägigen Organisationen und staatlichen Einrichtungen (bis auf die Bundespsychotherapeutenkammer) Standards, einheitliche Nomenklatur und Leitlinien für Psychosoziale Notfallversorgung (PSNV) festzulegen. An diesen Standards orientieren sich die Konferenz Evangelische NFS in der EKD, die Konferenz der Diözesanbeauftragten für die Katholische NFS, der Arbeiter-Samariter-Bund, das Deutsche Rote Kreuz, die Johanniter-Unfallhilfe, wenn sie 2013 Mindeststandards der Aus- und Fortbildung im Bereich der Psychosozialen Akuthilfe definieren (Müller-Lange et al. 2013: 437).

Der Mindestumfang beträgt 80 Unterrichtseinheiten und umfasst u.a. Grundlagen der Psychotraumatologie, Organisationskunde im Rettungsdienst und der Behörden 
für Ordnung und Sicherheit (BOS), Gesprächsführung, besondere Zielgruppen (Kinder, Menschen mit Behinderung, fremdsprachige Menschen). Besondere Einsatzlage mit spezieller Expertise (z.B. Tod im Betrieb, Suizid, Überbringung einer Todesnachricht), Recht und Verwaltung (Schweigepflicht!) wie auch Selbstschutz und Psychohygiene werden in allen Ausbildungsgängen der genannten Anbieter unterrichtet. Praxiserfahrung in Polizei, Feuerwehr und Rettungsdiensten in Form von Hospitationen ergänzt die Unterrichteinheiten. Regelmäßige Supervision und umfassende Dokumentation sind Teil der Qualitätssicherung.

NFS hat darüber hinaus Kurselemente wie „Theologische Grundlagen der NFS“, „Umgang mit Schuld“, „Gebet und Rituale“, als spezifische spirituelle und liturgische Kompetenzen, die NFS von weltlicher Akuthilfe unterscheiden. Besonderes Gewicht hat der Umgang mit den unterschiedlichen Religionszugehörigkeiten der betroffenen Opfer, Zeugen und Angehörigen - zeigen doch Forschungsergebnisse aus der Psychotraumatologie, dass Glaube und Zugehörigkeit zu Glaubensgemeinschaften zentrale Ressourcen zur Bewältigung extremen Erlebens darstellen. Die Vernachlässigung dieser Kräfte wäre allein aus fachlicher Hinsicht fahrlässig.

Angesichts von ca. 5\% muslimischem Bevölkerungsanteil sind Einsätze in muslimischen Familien Teil des Einsatzalltags der NFS. Die NFS hat in Zusammenarbeit mit den Organisationen der Muslime darauf reagiert, indem in der Ausbildung das „Krankheits- und Todesverständnis im Islam“ wesentlicher Unterrichtsgegenstand ist, indem eigene Ausbildungsgänge zur „muslimischen Notfallbegleitung“ unter Beteiligung muslimischer Theologen/innen erprobt werden (Müller-Lange et al. 2013: 500-504). Diese Fortbildungen sind an den o.a. „Gemeinsamen Standards zur Psychosozialen Akuthilfe" orientiert. Die Bedarfe und Lösungen werden nach den sehr unterschiedlichen regionalen Verhältnissen ausgerichtet. In der Regel wird angestrebt, dass zumindest die Nachalarmierung einer muslimischen Notfallbegleitung in jedem regionalen NFS-System möglich ist. Im Einsatzalltag zeigt sich, dass weniger die Barriere unterschiedlicher Glaubensrichtungen als die Sprachbarriere die größte Herausforderung für Einsatzkräfte und sicher auch Betroffene darstellt.

Die Zahl ordinierter Geistlicher in den NFS-Teams ist im Osten Deutschlands geringer als im Norden, Westen und Süden. Nichtordinierte psychosoziale Fachkräfte mit zusätzlich erworbener Seelsorgekompetenz werden aber in ganz Deutschland in den nächsten Jahren allein deshalb in größerer Zahl in den NFS-Systemen tätig sein, weil die Zahl der ordinierter Geistlichen insgesamt abnimmt.

\subsection{Umgang mit Sterben und Tod}

Sicherheit, Berechenbarkeit, umfassende Hilfs- und Rettungskompetenz umgeben die Menschen in Deutschland. Rauchmelder verhindern Erstickungstode in vielen Wohnungen, Gurtpflicht im PKW schafft passive Sicherheit bei Aufprall, ein gut funktionierendes Rettungssystem stellt exzellente Erstversorgung schon am Unfallort sicher.

NFS hat allenfalls mit ihrem Beitrag zu Schulung und Prävention für Einsatzkräfte teil an dieser Form von Gefahrenabwehr und Verhinderung von Traumatisierungen.

In der unmittelbaren Einsatzerfahrung aber geht es um die Bewältigung des Erlebens von Schrecken durch den jähen Tod. Tod ist der größte und hier in aller Regel völlig unerwartete „Störfall“. 
Seine Bewältigung kann so gut wie nie „trainiert“ werden. Stets überwiegen Hilflosigkeit und Fassungslosigkeit. NFS kann den Tod nicht rückgängig machen, sie kann allerdings mit der Grundkompetenz von Präsenz und Unterstützung durch das Angebot von Nähe die Opfer, Zeugen und Angehörigen begleiten, die vorübergehend desorientiert sind, erst nach und nach ihre eigenen Ressourcen wieder gewinnen werden. Dabei übt NFS keine Heilkunde aus, sie schützt in der Erstintervention auch vor dem Missverständnis, dass die Fülle von Symptomen (Steuerungsstörung, Dissoziation, emotionale Taubheit, hyperaktives Verhalten, vgl. Akute Belastungsstörung nach DSM IV 308.3) Zeichen einer seelischen Erkrankung darstellen. In der überwiegenden Zahl verschwinden diese Symptome in kurzer Frist (Müller-Cyran 2009: 38). Auch in Hinblick auf die Zeichen von Trauer weiß die NFS, dass die in der Seelsorgelehre umfassend reflektierten Erfordernisse der langfristigen Begleitung von Trauerprozessen ihren Ort keinesfalls in der Akutphase - also am Unfallort oder direkt nach Abbruch der Reanimationsmaßnahmen haben. So ist die Fachlichkeit der NFS im Zwischenraum zwischen später notwendigem heilkundlichen Regime angesichts möglicher seelischer Störungen wie Posttraumatische Belastungsstörung (PTBS) und Trauerseelsorge zu verorten. Beide benachbarten Fachexpertisen ersetzen keinesfalls die NFS.

Die Notfallseelsorgenden teilen mit Einsatzkräften und Angehörigen das Erleben von Hilfslosigkeit. Ihnen wird aber von diesen Sprachfähigkeit angesichts des Todes zugetraut und zugesprochen. Dieses Zutrauen wiederum ist eine essentielle Ressource der NFS. Ein Fundus an bewährter Sprache in Form der Jahrtausende alten Psalmen und die geprägte Sprache in Gebeten sind schon in der Entstehung deutlich auf Extremsituationen bezogen (Ps. 6, 3: „Herr, sei mir gnädig, denn ich bin schwach; heile mich, Herr, denn meine Gebeine sind erschrocken und meine Seele ist sehr erschrocken."). Der Notfallseelsorgende wird nun natürlich in der ersten Kontaktphase zunächst sich mit wenigen Worten vorstellen, seine Präsenz zusichern und Schweigen teilen können. Gebete finden eher am Ende einer Intervention ihren hilfreichen Platz. Im klar areligiösen Umfeld würden sie geradezu kontraindiziert sein.

Bewegend ist am Ende einer Intervention, wie oft das z.B. Angebot einer Aussegnung auch von Angehörigen gewünscht wird.

Ein Fallbeispiel kann erläutern, dass Hoffnung auf Wiedergewinnung der Sprachfähigkeit - in diesem Fall durch Feuerwehrkräfte - an den Notfallseelsorger gerichtet wird:

6:00 Auf einer eingleisigen Bahnstrecke umfährt ein PKW die geschlossene Halbschranke. Das Triebfahrzeug des Zuges erfasst den PKW und zieht ihn 150 Meter beim Bremsvorgang mit. Der PKW ist völlig zerquetscht als zerrissenes Blech unter dem Triebfahrzeug sichtbar. Den unverzüglich eintreffenden Rettungskräften aus Feuerwehr, Notärztin, Rettungssanitätern. Polizei und später Bundespolizei bleibt keine Möglichkeit zum rettenden oder wenigstens bergendem Handeln.

6:30 Der alarmierte NFS trifft ein, während die Fahrgäste zu einem Bus zur Weiterfahrt geführt werden. In der Supervision berichtet er später, dass der Anblick eines in den Trümmern sichtbaren Buggys für ihn und alle Kräfte das Belastendste war: jeder phantasiert ein totes Kind unter dem Zug ...

8:30 NFS hat seine eigene Ablösung nachalarmiert, da er zu einer Veranstaltung weiter muss. Er übergibt an seinen Hintergrunddienst-Kollegen. Währenddessen ist der Bergezug mit 
schwerem Hebezeug der DB AG unterwegs. Dies ist notwendig, da das regional vorhandene Hebezeug von Feuerwehr und THW nicht zur Hebung des Triebfahrzeugs am Zug ausreicht. In der späteren Supervision beschreibt der zweite NFS wie sein Kollege, dass das stundenlange erzwungene „Nichtstun“ neben den phantasierten Bildern des Schreckens (wer ist in dem Auto? Wie zerstört sind die Körper?) eigentlich die größte Last war. Die Feuerwehrleute beschreiben es in der Nachbesprechung in der Wache exakt genauso.

11:00 Der Bergezug ist eingetroffen, alles montiert, die Bergung kann beginnen. Der Einsatzleiter der Feuerwehr stellt die „Erste Linie“ von Kräften zusammen, die den erwarteten Anblick der Toten aushalten müssen und diese dann bergen sollen. Er bittet den NFS: „Sprechen Sie mit den Männern!" Der NFS berichtet in der Supervision von Hilflosigkeit in den ersten Sekunden („Was soll ich bloß sagen?“) und der beruhigenden Erfahrung, dass er mit wenigen Worten dann doch einige Worte der Ermutigung für die Feuerwehrkameraden gefunden hat - selber überrascht über die ihm zufallende Sprachfähigkeit.

Erstintervention bei Todesfällen erfordert eine klare zeitliche Perspektive. Als Einmalintervention muss der rückblickende Teil zu Beginn (Was ist geschehen? Wer war der Verstorbene für Sie?) übergeleitet werden in vorausschauende Erkundungen. Wer sollte nun an der Seite der Hinterbliebenen sein? Was ist als nächste zu tun? Wer könnte die Bestattung ausrichten? NFS ist für die Organisation ihrer eigenen Ablösung verantwortlich (Müller-Cyran 2009: 43). Manchmal wird durch Hinterbliebene der Wunsch geäußert, der Notfallseelsorger möge selbst die Bestattung übernehmen. Dies ist eigentlich ein gutes Zeichen, wenn man es als Signal für entstandene Nähe versteht. Dennoch muss solch ein Wunsch nach bleibendem Kontakt abgelehnt werden und der Kontakt in die gemeindliche Seelsorge angebahnt werden. Notfallseelsorge ist in aller Regel eine singuläre Intervention, die streng subsidiär für andere Fachdienste der „Zweiten Linie“ eintritt.

Dadurch verzichten die Mitarbeitenden der NFS auf die Rückmeldung über Erfolg ihrer Arbeit.

Ähnlichen Verzicht gibt es im Bereich der Telefonseelsorge. Andere psychosoziale und psychotherapeutische Dienste mit prozessorientierten Mehrfachinterventionen haben es - zumindest bei diesem Thema der intrinsischen Gratifikation durch fachliche Erfolge - leichter. Umso wichtiger ist ausreichende und qualifizierte Supervision für die Notfallseelsorgenden.

\section{Literatur}

APA (American Psychiatric Association) (1996) Diagnostisches und statistisches Manual psychischer Störungen. DSM-IV. 4. Aufl. (dt. Bearb. v. Saß H, Wittchen H-U, Zaudig M). Hogrefe, Göttingen

Gerngroß I (2015) Notfallpsychologie und psychologisches Krisenmanagement. Schattauer, Stuttgart

Lasogga F, Gasch B (2009) Psychische Erste Hilfe bei Unfällen. 4. Aufl. Stumpf + Kossendey, Edewecht

Lasogga F, Münker-Kramer E (2009) Psychosoziale Notfallhilfe. Stumpf + Kossendey, Edewecht

Müller-Cyran A (2009) Krisenintervention bei Menschen nach der Konfrontation mit dem plötzlichen Tod - die peritraumatische Krisenintervention. In: Bronisch T, Sulz S (Hrsg.) Krisenintervention und Notfall in Psychotherapie und Psychiatrie. 1. Aufl. CIP-Medien, München, S. 37-45

Müller-Lange J, Rieske U, Unruh I (Hg.) (2013) Handbuch Notfallseelsorge. Stumpf + Kossendey, Edewecht 


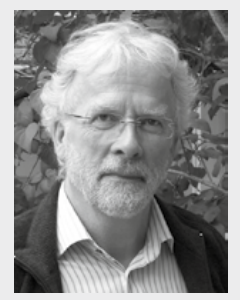

Pfarrer Christoph Pompe, Psychologischer Psychotherapeut

Studium der ev. Theologie und Psychologie in Bonn. Seit 1979 Leitung der evangelischen Beratungsstellen in Duisburg/Moers, in Oberhausen und ab 1995 in Detmold. Mentor der Telefonseelsorge Duisburg/Mülheim/Oberhausen. Leitung der Notfallseelsorge Lippe seit 1998. Mitglied des Vorstandes des Ev. Konferenz für Familien- und Lebensberatung (EKFuL) e.V. seit 1987. Fortbildung in Verhaltenstherapie, systemischer Familientherapie (Inst. für Familientherapie e.V. Weinheim), tiefenpsychologischer Paarberatung (Ev. Zentralinstitut Berlin), Psychodynamisch Integrativer Traumatherapie (PITT). Fachberater Seelsorge der Feuerwehr Lippe. 